\title{
Tree-Level Vacuum Stability in Multi Higgs Models
}

\author{
A. Barroso, Pedro Ferreira* tand R. Santos \\ CFTC, Universidade de Lisboa, Portugal \\ E-mail: barroso@cii.fc.ul.pt, ferreira@cii.fc.ul.pt, \\ rsantos@cii.fc.ul.pt
}

In the most general model with two Higgs doublets, if a minimum that preserves the $U(1)_{\text {em }}$ symmetry exists, then charge breaking (CB) minima cannot occur. The depth of the potential at a stationary point that breaks $\mathrm{CB}$ or $\mathrm{CP}$, relative to the $U(1)_{e m}$ preserving minimum, is proportional to the squared mass of the charged or pseudoscalar Higgs, respectively.

International Europhysics Conference on High Energy Physics

July 21st - 27th 2005

Lisboa, Portugal

\footnotetext{
* Speaker.

${ }^{\dagger}$ P.M.F. is supported by FCT under contract SFRH/BPD/5575/2001.
} 
In this talk we will review recent results $[1,2]$ on symmetry breaking in two Higgs doublet models (2HDM) [3]. Namely, the possibility that minima that break different symmetries can appear simultaneously in the potential, and tunneling between them might occur. In ref. [1] we worked in 2HDM without explicit CP breaking and showed that if there is, at tree level, a minimum that preserves the $U(1)_{e m}$ and CP symmetries, that minimum is the global one. Therefore, the stability of this minimum is guaranteed and tunneling to a deeper one that breaks charge conservation or CP becomes impossible. In ref. [2] we extended this analysis to the most general 2HDM and proved that, once a charge-preserving minimum exists, any charge breaking (CB) stationary point that might exist lies above the minimum. Charge conservation is thus assured.

There are many ways of writing the $2 \mathrm{HDM}$ tree-level potential, for this talk we will use the one introduced in ref. [4]. With two scalar Higgs doublets in the theory, $\Phi_{1}$ and $\Phi_{2}$, both having hypercharges $Y=1^{1}$,

$$
\Phi_{1}=\left(\begin{array}{c}
\varphi_{1}+i \varphi_{2} \\
\varphi_{5}+i \varphi_{7}
\end{array}\right), \Phi_{2}=\left(\begin{array}{c}
\varphi_{3}+i \varphi_{4} \\
\varphi_{6}+i \varphi_{8}
\end{array}\right)
$$

there are four $S U(2)_{W} \times U(1)_{Y}$ invariants one can construct with these fields, namely

$$
\begin{aligned}
x_{1} \equiv\left|\Phi_{1}\right|^{2} & =\varphi_{1}^{2}+\varphi_{2}^{2}+\varphi_{5}^{2}+\varphi_{7}^{2} \\
x_{2} \equiv\left|\Phi_{2}\right|^{2} & =\varphi_{3}^{2}+\varphi_{4}^{2}+\varphi_{6}^{2}+\varphi_{8}^{2} \\
x_{3} \equiv \operatorname{Re}\left(\Phi_{1}^{\dagger} \Phi_{2}\right) & =\varphi_{1} \varphi_{3}+\varphi_{2} \varphi_{4}+\varphi_{5} \varphi_{6}+\varphi_{7} \varphi_{8} \\
x_{4} \equiv \operatorname{Im}\left(\Phi_{1}^{\dagger} \Phi_{2}\right) & =\varphi_{1} \varphi_{4}-\varphi_{2} \varphi_{3}+\varphi_{5} \varphi_{8}-\varphi_{6} \varphi_{7} .
\end{aligned}
$$

Notice that under a particular CP transformation in this basis $\left(\Phi_{1} \rightarrow \Phi_{1}^{*}, \Phi_{2} \rightarrow \Phi_{2}^{*}\right)$ the invariants $x_{1}, x_{2}$ and $x_{3}$ remain the same but $x_{4}$ changes sign. The most general tree-level potential is thus composed of all the terms linear and quadratic in the $x$ 's, i.e.,

$$
\begin{aligned}
V= & a_{1} x_{1}+a_{2} x_{2}+a_{3} x_{3}+a_{4} x_{4}+b_{11} x_{1}^{2}+b_{22} x_{2}^{2}+b_{33} x_{3}^{2}+b_{44} x_{4}^{2}+ \\
& b_{12} x_{1} x_{2}+b_{13} x_{1} x_{3}+b_{14} x_{1} x_{4}+b_{23} x_{2} x_{3}+b_{24} x_{2} x_{4}+b_{34} x_{3} x_{4} .
\end{aligned}
$$

The $a_{i}$ parameters have dimensions of mass squared and the $b_{i j}$ parameters are dimensionless. The potential thus written depends on 14 real parameters but, with a particular choice of basis, one can reduce this number to 11 independent parameters (see, for instance, [5]). The linear terms in $x_{4}$ are the ones that explicitly break $\mathrm{CP}$, and if we eliminate them we are left with the 10 parameter $\mathrm{CP}$ preserving potential that was used in ref. [1] (with a judicious choice of basis [5] the number of independent real parameters of this potential may be reduced to 9).

It is a well known fact [3] that the 2HDM potential can only have three types of minima. One of them is a charge breaking minimum where three of the fields, at least one of them carrying electrical charge, have non-vanishing vacuum expectation values (vevs). For instance, the fields $\varphi_{5}, \varphi_{6}$ and $\varphi_{3}$. In the second possible type of minimum only neutral fields have vevs and there are two possibilities. In the first only two fields have vevs ( $\varphi_{5}$ and $\varphi_{6}$, for instance), and we call this the first "normal" minimum, $N_{1}$. In the second case there are three vevs, for the fields $\varphi_{5}, \varphi_{6}$

\footnotetext{
${ }^{1}$ The numbering of the real scalar $\varphi$ fields is chosen for convenience of writing the mass matrices for the scalar particles.
} 
and $\varphi_{7}$, for example. We call this case the $N_{2}$ minimum. Notice that when the model is reduced to the potential that explicitly preserves $\mathrm{CP}$, the $N_{2}$ minimum spontaneously breaks $\mathrm{CP}$. For this more general case, however, there is a priori no physical distinction between the two "normal" minima, both of them preserving charge conservation.

In references [1] and [2] we developed a method to compute the value of the tree-level potential at each of these stationary points, and compare their value. We refer the readers to those publications for details of the calculations, and proceed to present the results and their consequences. For the $N_{1}$ minimum, the vevs will be $\varphi_{5}=v_{1}$ and $\varphi_{6}=v_{2}$; for $\mathrm{CB}$, we will have $\varphi_{5}=v_{1}^{\prime}, \varphi_{6}=v_{2}^{\prime}$ and $\varphi_{3}=\alpha$ (this last vev is the charged one that breaks charge conservation); for $N_{2}, \varphi_{5}=v_{1}^{\prime \prime}, \varphi_{6}=v_{2}^{\prime \prime}$ and $\varphi_{7}=\delta$ (in the case of the CP preserving potential, this last vev is the one that spontaneously breaks $\mathrm{CP}$ ). The difference between the values of the potential at a $\mathrm{CB}$ and an $N_{1}$ stationary points is given by

$$
V_{C B}-V_{N_{1}}=\frac{1}{2} Y^{T} V^{\prime}=\frac{M_{H^{ \pm}}^{2}}{2 v^{2}}\left[\left(v_{1}^{\prime} v_{2}-v_{2}^{\prime} v_{1}\right)^{2}+\alpha^{2} v_{1}^{2}\right]
$$

where $M_{H^{ \pm}}^{2}$ is the value of the squared charged scalar mass at $N_{1}$. Then, if $N_{1}$ is a minimum, we will necessarily have $M_{H^{ \pm}}^{2}>0$ and, given that the quantity in square brackets above is always positive, we conclude that $V_{C B}-V_{N_{1}}>0$. Then, the CB stationary point is clearly above the $N_{1}$ minimum. Furthermore, it is possible to show that under these circumstances the matrix of CB squared scalar masses is neither positive nor negative definite. As a result, we reach the conclusion the the $\mathrm{CB}$ stationary point is a saddle point, and lies above the $N_{1}$ minimum.

Results altogether identical are obtained if one compares the $N_{2}$ and CB potentials. From [2] we see that

$$
V_{C B}-V_{N_{2}}=\left(\frac{M_{H^{ \pm}}^{2}}{2 v^{2}}\right)_{N_{2}}\left[\left(v_{1}^{\prime} v_{2}^{\prime \prime}-v_{2}^{\prime} v_{1}^{\prime \prime}\right)^{2}+\alpha^{2}\left(v_{1}^{\prime \prime 2}+\delta^{2}\right)+\delta^{2} v_{2}^{\prime 2}\right],
$$

where now $\left(M_{H^{ \pm}}^{2}\right)_{N_{2}}$ is the squared charged scalar mass of the $N_{2}$ stationary point, and $\left(v^{2}\right)_{N_{2}}=$ $v^{\prime \prime 2}+v_{1}^{\prime \prime 2}{ }_{2}+\delta^{2}$. Again, we reach the conclusion that, if $N_{2}$ is a minimum, then $V_{C B}-V_{N_{2}}>0$, and the $\mathrm{CB}$ stationary point lies above the normal minimum, Again, it is possible to demonstrate that the CB stationary point is a saddle point. The conclusion to take from this analysis is that, if a minimum that preserves electric charge conservation exists, it is necessarily deeper than any $\mathrm{CB}$ stationary point that might exist in the model. Further, that stationary point is necessarily a saddle point. There is therefore no possibility whatsoever of tunneling from a charge-preserving minimum to a deeper one where charge is broken, and the masslessness of the photon is thus assured.

What about a comparison between the values of the potential at $N_{1}$ and $N_{2}$ stationary points? Unfortunately we cannot reach any definite conclusion about which of these possible minima is deeper. Following a chain of thought altogether identical to the previous cases, one obtains

$$
V_{N_{2}}-V_{N_{1}}=\frac{1}{2}\left[\left(\frac{M_{H^{ \pm}}^{2}}{v^{2}}\right)_{N_{1}}-\left(\frac{M_{H^{ \pm}}^{2}}{v^{2}}\right)_{N_{2}}\right]\left[\left(v_{1}^{\prime \prime} v_{2}-v_{2}^{\prime \prime} v_{1}\right)^{2}+\delta^{2} v_{2}^{2}\right]
$$

Depending on which stationary point has a larger value for the squared charged mass, then, either $N_{1}$ or $N_{2}$ might be deeper. This seems to depend on the particular values of the parameters of the model, both cases a priori possible. A very interesting thing happens, though, when we restrict 
ourselves to the case of the $\mathrm{CP}$ preserving potential. In that case the $N_{1}$ minimum preserves both electric charge conservation and $\mathrm{CP}$, and the $N_{2}$ stationary point spontaneously breaks CP. Calling $V_{N_{1}}=V_{N}$ and $V_{N_{2}}=V_{C P}$, and investigating the mass matrices of the 2HDM model (for instance, [1]) we obtain a remarkable result,

$$
V_{C P}-V_{N}=\frac{M_{A}^{2}}{2 v^{2}}\left[\left(v_{1}^{\prime \prime} v_{2}-v_{2}^{\prime \prime} v_{1}\right)^{2}+\delta^{2} v_{2}^{2}\right]
$$

where $M_{A}^{2}$ is, as usual, the squared pseudoscalar mass at the normal (i.e., charge and CP preserving) minimum. The right-hand side of this equation is thus positive and we have, just as in the CB case, $V_{C P}-V_{N}>0$. The CP stationary point is therefore above the normal minimum, but in this case it is not obvious whether it is also a saddle point. Thus no tunneling to a deeper minimum may occur once the potential is at a vacuum that respects both CP and charge conservation. The tree level vacuum, we have therefore shown, is perfectly stable.

An intriguing aspect of these results is the following: if one observes equations (4) and (5), one sees that the difference in the depth of the potential between the normal minimum and the CB stationary point is "controlled" by the charged Higgs squared mass. On the other hand, equation (7) shows that the difference in the value of the potential between the $\mathrm{CP}$ and the normal stationary points is proportional to the pseudoscalar squared mass. That is, the depth of the potential at a stationary point that breaks a given symmetry, relative to the normal minimum, depends, in a very straightforward manner, on the mass of the scalar particle directly linked with that symmetry. The absence of charge breaking when normal minima exist seems to be related to the non-existence, in the potential, of cubic terms in the fields. In fact, analysing the Zee model [6] scalar potential - this model consists of the $2 \mathrm{HDM}$ plus a charged $S U(2)$ singlet scalar -, where such terms are present, CB minima deeper than the normal ones are discovered [7]. This is not surprising, since charge - and colour - breaking is known to occur in supersymmetric theories [8], for which the scalar potential has, once again, cubic terms in the fields.

\section{References}

[1] P.M. Ferreira, R. Santos and A. Barroso, Phys. Lett. B603 (2004) 219; Erratum, Phys. Lett. B629 (2005) 114.

[2] P.M. Ferreira, R. Santos and A. Barroso, to be published in Phys. Lett. B; [hep-ph/ 0507224$]$

[3] T.D. Lee, Phys. Rev. D8 (1973) 1226;

G.C. Branco, Phys. Rev. D22 (1980) 2901;

M. Sher, Phys. Rep. 179 (1989) 273;

G.C. Branco, L. Lavoura and J.P. Silva, CP Violation, Oxford University Press, Oxford, England 1999.

[4] J. Velhinho, R. Santos e A. Barroso, Phys. Lett. B322 (1994) 213.

[5] S. Davidson and H.E. Haber, Phys. Rev. D72 (2005) 035004; J.F. Gunion and H.E. Haber, Phys.Rev. D72 (2005) 095002.

[6] A. Zee, Phys. Lett. B93 (1980) 389.

[7] A. Barroso and P.M. Ferreira, Phys. Rev. D72 (2005) 075010.

[8] J.M. Frére, D.R.T. Jones and S. Raby, Nucl. Phys. B222 (1983) 11. 